\title{
Square Maxwell's fish-eye lens for near-field broadband achromatic super-resolution imaging
}

\author{
Jue Li (李 珏 $)^{1,2}$, Yangyang Zhou (周杨阳) $)^{1,2^{*}}$, and Huanyang Chen (陈焕阳) $)^{1,2^{* *}}$ \\ ${ }^{1}$ Institute of Electromagnetics and Acoustics and Department of Physics, Xiamen University, Xiamen 361005, China \\ ${ }^{2}$ Fujian Engineering Research Center for EDA, Fujian Provincial Key Laboratory of Electromagnetic Wave Science and Detection Technology, \\ Xiamen Key Laboratory of Multiphysics Electronic Information, Xiamen University, Xiamen 361005, China \\ *Corresponding author: zhouyangyang@stu.xmu.edu.cn \\ ${ }^{*}$ Corresponding author: kenyon@xmu.edu.cn \\ Received October 25, 2021 | Accepted December 13, 2021 | Posted Online January 4, 2022
}

\begin{abstract}
Broadband super-resolution imaging is important in the optical field. To achieve super-resolution imaging, various lenses from a superlens to a solid immersion lens have been designed and fabricated in recent years. However, the imaging is unsatisfactory due to low work efficiency and narrow band. In this work, we propose a solid immersion square Maxwell's fish-eye lens, which realizes broadband (7-16 GHz) achromatic super-resolution imaging with full width at half-maximum around $0.2 \lambda$ based on transformation optics at microwave frequencies. In addition, a super-resolution information transmission channel is also designed to realize long-distance multi-source super-resolution information transmission based on the super-resolution lens. With the development of 3D printing technology, the solid immersion Maxwell's fish-eye lens is expected to be fabricated in the high-frequency band.
\end{abstract}

Keywords: solid immersion square Maxwell's fish-eye lens; super-resolution imaging; conformal mapping.

DOI: 10.3788/COL202220.031101

\section{Introduction}

Microscopy is a significant tool for research on life science and natural science. The resolution of the conventional lens system is constrained above one half-wavelength, due to the diffraction limit that evanescent waves carrying small amounts of information of an object decay in the far field. Many efforts have been made to overcome the diffraction limit; one important step is the proposed perfect lens in $2000^{[1]}$. The perfect lens can achieve perfect imaging by negative refractive materials to restore amplitude of all evanescent waves and phase of all propagating waves at the imaging plane. Following this idea, a series of superlenses ${ }^{[2-4]}$ were designed from the microwave to optical band ${ }^{[5,6]}$. Later, the hyperlens as a far-field magnified superresolution lens was proposed by hyperbolic dispersion materials, which can transform evanescent waves into far-field propagating waves. Utilizing alternative metal and dielectric materials, a one-dimensional hyperlens ${ }^{[7]}$ and a two-dimensional hyperlens ${ }^{[8]}$ have been designed and fabricated. However, for the superlens and hyperlens, it is difficult to push forward to applications, and relative experimental works were rarely reported in recent years due to the challenge of fabrication and the large loss from plasmonic resonance mechanisms and impedance mismatching between the object and lens.
On the other hand, utilizing total internal reflection (TIR) happening at the interface to excite evanescent waves, solid immersion lenses (SILs) ${ }^{[9,10]}$ were suggested to overcome the diffraction limit for super-resolution imaging. They have been studied widely through the application of high refractive-index (RI) solid material and specific geometric optical design ${ }^{[1-13]}$. With the development of materials sciences, different types of SILs were designed and fabricated from conventional structures to novel metamaterials structures ${ }^{[14-16]}$. However, a major drawback of SILs is their narrow band imaging capability due to aberration.

Recently, absolute instruments with a gradient RI (GRIN) profile ${ }^{[17-24]}$ have been developed rapidly for their excellent capability to control the propagation of electromagnetic waves ${ }^{[25-30]}$ and especially geometrical perfect imaging. Among these absolute instruments, Maxwell's fish-eye lens $(\mathrm{MFEL})^{[31]}$ has attracted a great amount of attention due to its properties of geometrical perfect imaging optics and large numerical aperture (NA). Many applications, based on the MFELs, were designed and fabricated from the microwave frequency to optical frequency ${ }^{[32]}$. Significantly, combining the solid immersion mechanism with geometrical perfect imaging of MFEL, a solid immersion MFEL as a near-field super-resolution lens ${ }^{[33,34]}$ has been designed and fabricated for super-resolution 
imaging at microwave frequencies. However, due to imaging along the lens's curved surface, there is imaging distortion, which limits the structure shape of the imaging object for further applications.

In this work, we utilize power tool transformation optics $(\mathrm{TO})^{[35-38]}$ to design a solid immersion square MFEL, which realizes super-resolution imaging and overcomes the disadvantage of the solid immersion circular MFEL. This paves the way for further applications of solid immersion MFEL. Utilizing SchwartzChristoffel (SC) mapping ${ }^{[39]}$, the solid immersion circular MFEL is transformed into a solid immersion square MFEL to achieve a wide-band (7-16 GHz) high-resolution imaging effect with FWHM around $0.2 \lambda$ as well as NA of one. With the commercial software COMSOL Multiphysics, we numerically calculate the functionalities of the lens and prove the excellent imaging ability of high resolution and wide band for the proposed solid immersion square MFEL.

\section{Square MFEL for High Resolution}

Let us recall an MFEL with a GRIN distribution that can realize perfect imaging. Assuming an MFEL located in virtual space ( $w$ space), its RI distribution can be expressed as follows ${ }^{[40]}$ :

$$
n_{w}=\frac{2 n_{0}}{1+\left(\frac{r_{w}}{R}\right)^{2}},
$$

where $r_{w}$ is the distance from the center of the lens. $R$ is the radius of the lens, $2 n_{0}$ denotes the RI at the center of MFEL, and $n_{0}$ represents the ambient RI. Figure 1 (c) shows the RI distribution of MFEL, where the lens radius is one (the unit is arbitrary), and $n_{0}$ is set to one. The MFEL can realize aberration-free imaging from point to point in the lens ${ }^{[22]}$, as shown in Fig. 1(c). It also can achieve super-resolution imaging in wave optics via introducing a solid immersion mechanism ${ }^{[34]}$. Although superresolution imaging can be achieved through solid immersion MFEL, its application scope is limited due to the curved boundary of the lens, leading to distortion of the imaging. To overcome imaging distortion due to the curved boundary of MFEL and extend the application prospects, the curved edge of the MFEL is considered to be changed into a straight edge according to the theory of conformal mapping. In this way, the image and object points are on the plane, which can better maintain and transmit the original shape. Such design of a solid immersion square MFEL is obtained based on the solid immersion circular MFEL by utilizing SC mapping ${ }^{[39]}$ as follows:

$$
\begin{gathered}
q(z)=1-\frac{1}{\operatorname{sn}\left\{\frac{2 i \sqrt{2} \pi^{3 / 2}[z+(1-i)]}{\Gamma\left(-\frac{1}{2}\right)^{2}} \mid 2\right\}^{2}}, \\
\omega(q)=\frac{q(z)-i}{q(z)+i},
\end{gathered}
$$

where $\operatorname{sn}(\varphi \mid m)$ represents the Jacobian elliptic function with the modulus of the ellipse $m$, and $\Gamma$ is the gamma function.

According to Eq. (2), we choose a transformation from the upper half-plane (coordinates $q$ ) to the square in physical space (coordinates $z$ ). Another conformal mapping, which is from the unit disk (coordinates $w$ ) to the upper half-plane (coordinates q), can be implemented based on Eq. (3). Consequently, an SC transformation from the unit disk to the square can be accomplished by taking the two equations together, which is the conformal mapping from a circular MFEL onto the square MFEL. The transformation relationship can be easily seen in Figs. 1(a) and 1(b), where the orthogonal mesh in the constant radial and azimuthal coordinates ( $r-\theta$ directions) within a unit disk in Fig. 1(a) is transformed into the orthogonal mesh inside the square in Fig. 1(b) by the SC mapping. Thus, the transformed RI distribution in physical space can be obtained as follows:

$$
n_{z}=n_{w}\left|\frac{\mathrm{d} w}{\mathrm{~d} z}\right| .
$$

Here, the RI distribution of the square MEFL is calculated by Eq. (4) based on a circular MFEL with $n_{0}=1$ and $n_{0}=2.4$, as shown in Figs. 1(d) and 1(e). It can be seen that the square MFEL achieves aberration-free imaging. According to the theory of TO, it can be inferred that the solid immersion square MFEL can also achieve super-resolution imaging, which is similar to the solid immersion circular MFEL.

To analyze the influence of the TIR mechanism for superresolution effects in the solid immersion square MFEL, the (a)

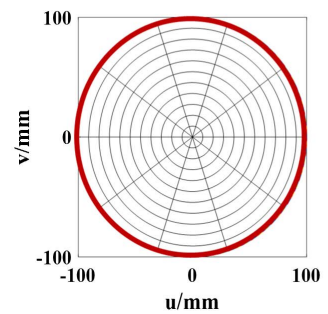

(b)

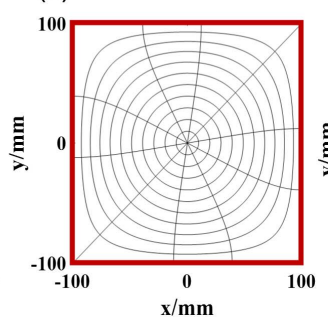

(c)

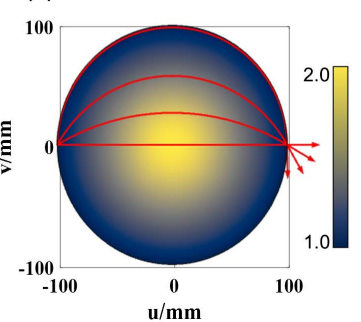

(d)

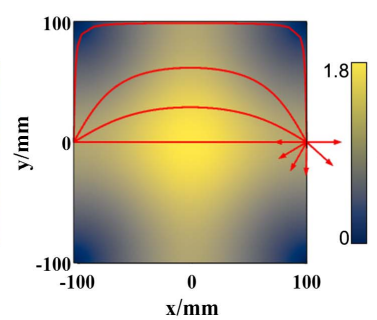

(e)

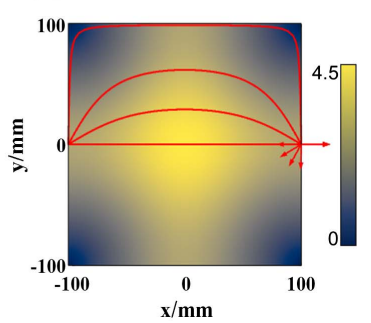

Fig. 1. (a) Coordination mapping in virtual space (w coordinate). (b) Coordination mapping in physical space (z coordinate). (c) The RI distribution of MFEL in virtual space (w coordinate) and ray trajectories from a point light source. (d), (e) The RI distribution of the square MFEL according to SC mapping in physical space ( $z$ coordinate) based on MFEL with $n_{0}=1$ and $n_{0}=2.4$, respectively, and the corresponding ray trajectories from a point light source. 
value $n_{0}$ is artificially changed from 1 to 3.1 at an interval of 0.7 to explore the relationship between the value of $n_{0}$ and the imaging effect of the square MFEL, as shown in Figs. 2(a) -2 (d). The focusing effect of the square MFEL with $n_{0}$ of $1,1.7,2.4$, and 3.1 at the frequency of $15 \mathrm{GHz}$ is simulated by method of full-wave numerical simulation, respectively. A point source [or a line current source for transverse electric (TE) polarization of light] is placed at $x=-100 \mathrm{~mm}$ and $y=0 \mathrm{~mm}$ (the center of the square MFEL is at the origin) as an extinction source. In the figure, there is a sub-wavelength light spot at the corresponding point of the square MFEL. The white dotted line represents the observation position of the imaging point $(x=101 \mathrm{~mm}, y=-100$ to $100 \mathrm{~mm}$ ), which is not at the boundary of the square MFEL but in the air near the boundary of the lens, and the NA of the square MFEL satisfies NA $=n \sin \theta=n_{\text {air }} \sin 90^{\circ}=1$. The red curve is the normalized electric field intensity of the imaging point, and the corresponding FWHM is calculated. It can be observed from Figs. 2(a)-2(d) that with the increase of the value of parameter $n_{0}$, the TIR phenomenon at the interface between the square MFEL and the air is gradually enhanced. Therefore, the evanescent wave is excited by the TIR mechanism at the interface of the lens/air, and the values of the corresponding FWHM at the imaging point gradually decrease from $0.34 \lambda$ to $0.16 \lambda$, which is much less than the diffraction limit $0.51 \lambda$. This manifests that the solid immersion square MFEL also has excellent super-resolution imaging capabilities and can achieve the same effect as the solid immersion circular MFEL. The relative electric field patterns are shown in Figs. 2(e)-2(h), which also well explain the good imaging performance of the square MFEL due to the almost completely axisymmetric field distribution. Consequently, through the above analysis, it is noted that with the increase of $n_{0}$, the super-resolution imaging effect of the square MFEL is gradually improved by the TIR mechanism.

Next, we will discuss the frequency response of the super-resolution effect in the square MFEL. A square MFEL with $n_{0}=2.4$ is selected for analyzing the broadband imaging performance of the solid immersion square MFEL at different frequencies from $7 \mathrm{GHz}$ to $16 \mathrm{GHz}$. A point source is placed at $x=-100 \mathrm{~mm}$ and $y=0 \mathrm{~mm}$ to excite the cylindrical TE wave. The imaging effects of the solid immersion square MEFLs at different frequencies are observed at $x=101 \mathrm{~mm}$ and $y=-100$ to $100 \mathrm{~mm}$ (located at the air background), so the NA of the square MFEL is one. Figures 3(a)-3(d) illustrate the electric field intensity $\left|E^{2}\right|$ and the corresponding FWHM of the solid immersion square MFELs at frequencies of $7 \mathrm{GHz}, 10 \mathrm{GHz}, 13 \mathrm{GHz}$, and $16 \mathrm{GHz}$, respectively. It can be seen that the corresponding FWHMs at frequencies of $7 \mathrm{GHz}, 10 \mathrm{GHz}, 13 \mathrm{GHz}$, and $16 \mathrm{GHz}$ are all less than $0.25 \lambda$, which is far below the diffraction limit. From the figures, it is noted that part of super-resolution imaging is located at the air background, and a slight resonance emerges in the lens compressing the resolution. At the same time, Figs. 3(e)-3(h) show the corresponding electric field distribution patterns. From the above analysis, it is found that the solid immersion square MFEL can achieve super-resolution imaging at most frequencies. However, the super-resolution imaging is invalid at other specific frequencies due to the destruction from stable whispering gallery mode $(\mathrm{WGM})^{[34]}$, which is a resonance mode due to continuous TIR of the impedance mismatching at the interface of lens/air.

To circumvent the disadvantage of WGMs, which are easily generated due to the four corners, we optimize the design and improve the resolution of solid immersion MFEL, and propose (a)

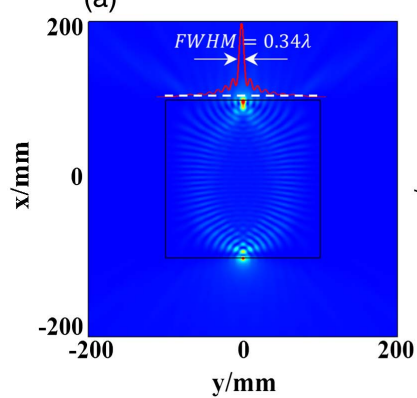

(e)

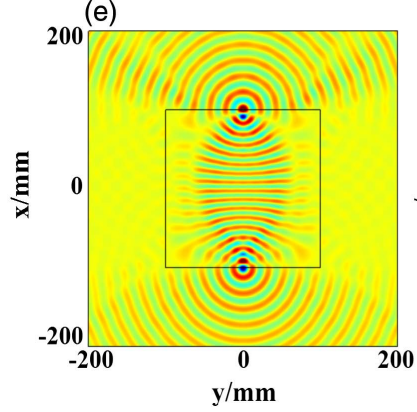

(b)
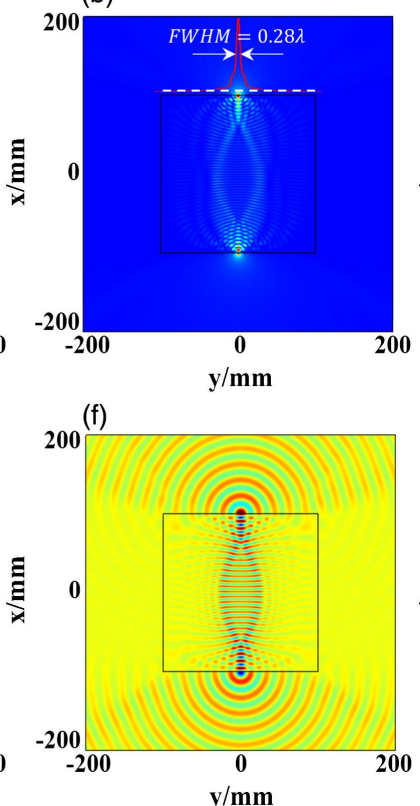

(c)
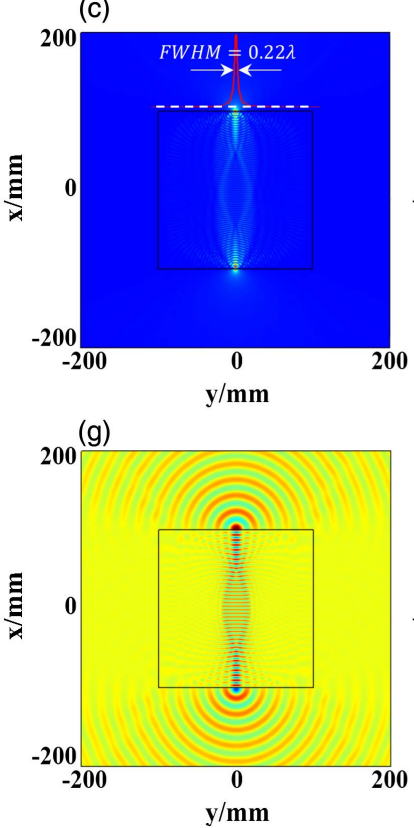

(d)

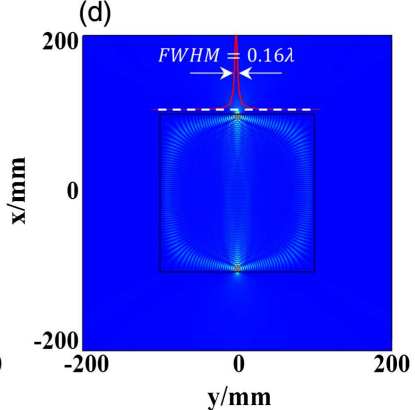

(h)

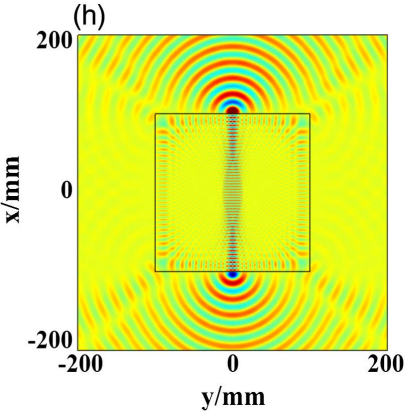

Fig. 2. Imaging performance of the square MFEL with different $n_{0}$. (a)-(d) The related electric field intensity distribution and the corresponding FWHM of the square MFEL at $15 \mathrm{GHz}$ with $n_{0}$ of 1, 1.7, 2.4, and 3.1, respectively. (e)-(h) The relative electric field $E_{z}$ distributions, respectively. 
(a)

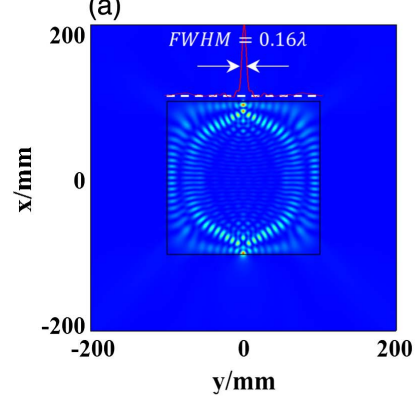

(e)

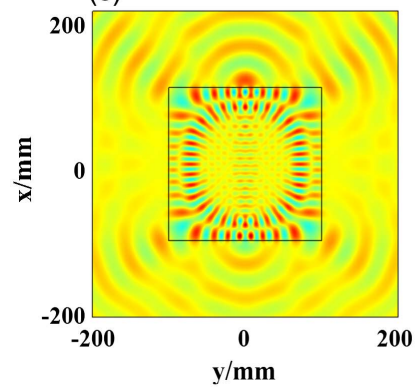

(b)

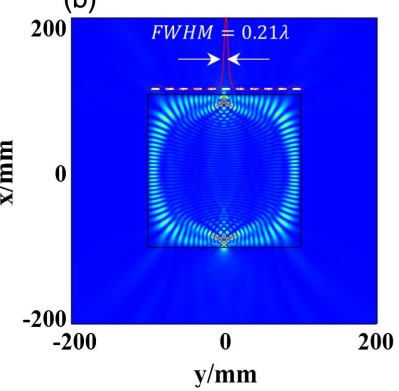

(f)

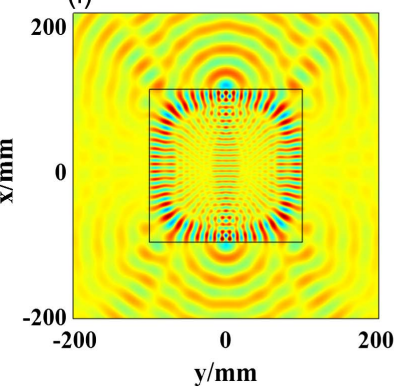

(c)

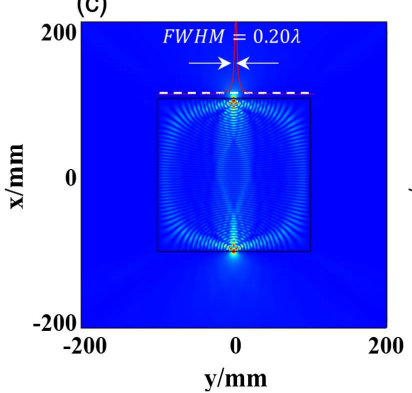

(g)

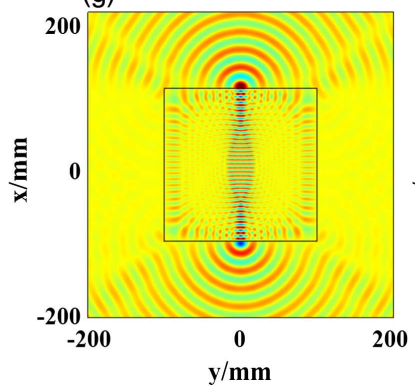

(d)

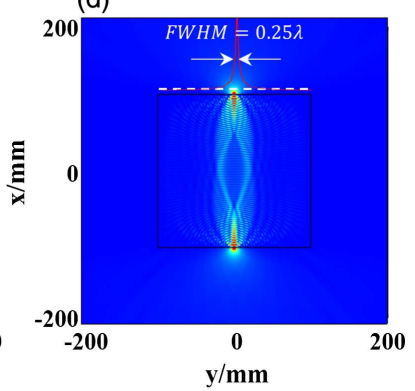

(h)

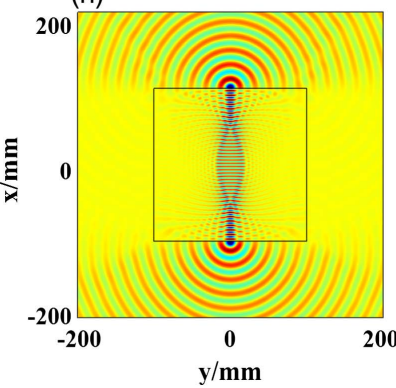

Fig. 3. Broadband imaging effect of solid immersion square MFEL at different frequencies. (a)-(d) The electric field intensity and the corresponding FWHM of the solid immersion square MFEL at frequencies of $7 \mathrm{GHz}, 10 \mathrm{GHz}, 13 \mathrm{GHz}$, and $16 \mathrm{GHz}$, respectively. (e)-(h) The corresponding real part of electric field distribution, respectively.

a new quasi-square MFEL transformed from the circular MFEL by the method of SC mapping, as shown in Fig. 4(d). The quasisquare MFEL avoids the disadvantage caused by the right angle of a square MFEL, mainly through bending the four angles from straight to curved. Next, we will numerically calculate the imaging performance of the quasi-square MFEL and square MFEL (a)

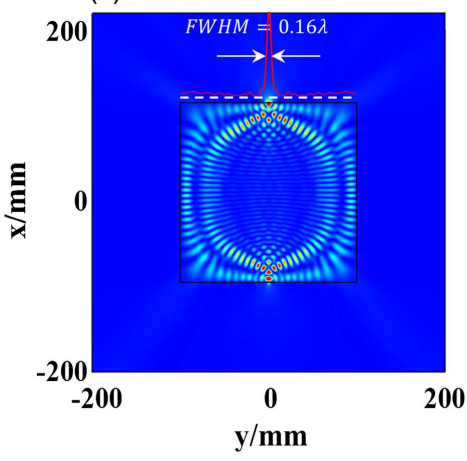

(d)

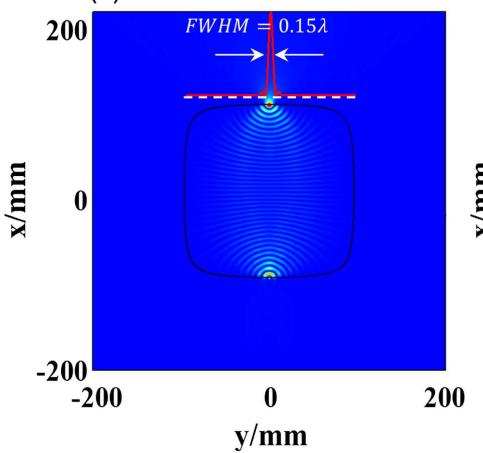

(b)

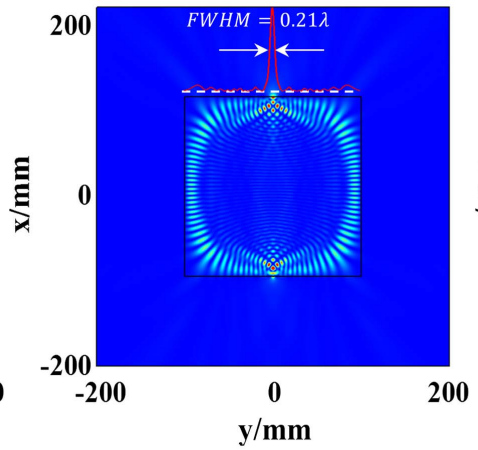

(e)

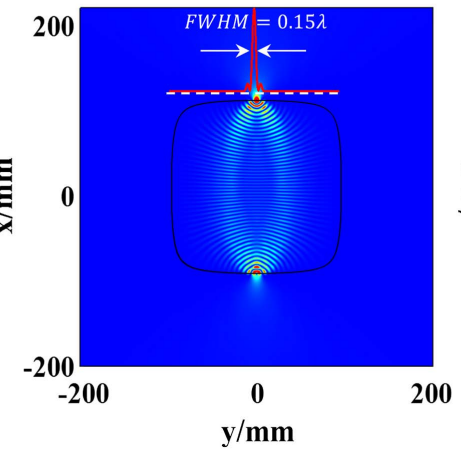

(c)

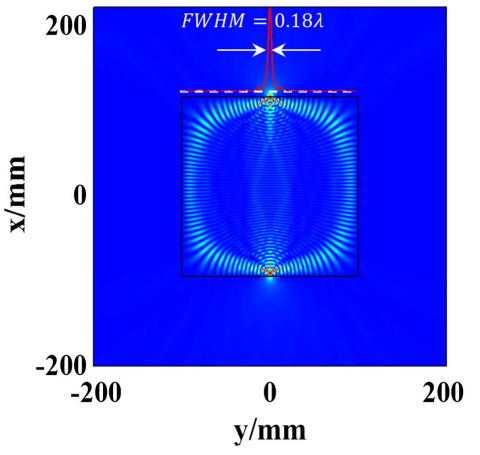

(f)

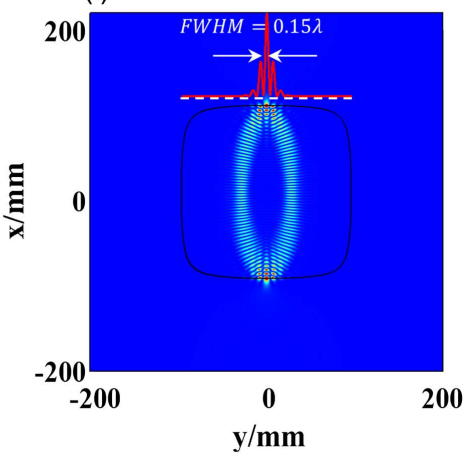

Fig. 4. (a)-(f) Electric field intensity distribution and the corresponding FWHM of the solid immersion square MFEL (the first row) and solid immersion quasi-square MFEL (the second row) at frequencies of $8 \mathrm{GHz}$, $10 \mathrm{GHz}$, and $12 \mathrm{GHz}$, respectively. 
with $n_{0}=2.4$, where a point source is placed at $x=-100 \mathrm{~mm}$ and $y=0 \mathrm{~mm}$ as an exciting source. Figures $4(\mathrm{~d})-4(\mathrm{f})$ show the electric field intensity and the corresponding FWHM of the solid immersion quasi-square MFEL at frequencies of $8 \mathrm{GHz}$, $10 \mathrm{GHz}$, and $12 \mathrm{GHz}$, respectively. To better illustrate the optimized imaging effect, the electric field intensity and the corresponding FWHM of the solid immersion square MFEL at frequencies of $8 \mathrm{GHz}, 10 \mathrm{GHz}$, and $12 \mathrm{GHz}$ are also shown in Figs. 4(a)-4(c). By comparing their electric field intensity distribution, it can be found that the solid immersion quasisquare MFEL significantly avoids distribution of the WGM resonance, and only symmetrical focusing imaging is formed. Consequently, the solid immersion quasi-square MFEL surmounts the disadvantage and achieves better resolution than the solid immersion square MFEL. It is proved that the quasi-square MFELs are further optimized in the super-resolution effect. However, the broadband effect of the quasi-square MFELs is not as good as that of the square MFEL, and it is more difficult to achieve when considering the experiments. Therefore, the square MFEL is mainly discussed here.

Furthermore, to better illustrate the super-resolution effect of solid immersion MFELs, the imaging performances of a solid immersion square MFEL with $n_{0}=2.4$ and a conventional square MFEL with $n_{0}=1$ are compared with two exciting point sources placed with a distance of spacing $\lambda / 3$ at the frequencies of $8 \mathrm{GHz}, 9 \mathrm{GHz}, 11 \mathrm{GHz}$, and $12 \mathrm{GHz}$, respectively, as shown in Fig. 5. The relative distribution curve of electric field intensity in air is calculated, and the imaging quality of square MFELs for two different $n_{0}$ is analyzed. Figures $5(\mathrm{e})-5(\mathrm{~h})$ reveal that the conventional square MFELs with $n_{0}=1$ fail to resolve the two points with spacing less than half wavelength, and only

one focal point is presented from the electric field intensity curve (red curve) at the frequencies of $8 \mathrm{GHz}, 9 \mathrm{GHz}, 11 \mathrm{GHz}$, and $12 \mathrm{GHz}$, respectively. In other words, two image points cannot be distinguished, indicating that the resolution of conventional square MFELs with $n_{0}=1$ is not enough. Oppositely, the square MFELs with $n_{0}=2.4$ can resolve two image points at frequencies of $8 \mathrm{GHz}, 9 \mathrm{GHz}, 11 \mathrm{GHz}$, and $12 \mathrm{GHz}$, respectively, as shown in Figs. 5(a)-5(d). The relative electric field intensity curve at the imaging plane also reveals that the solid immersion square MFEL with $n_{0}=2.4$ has a super-resolution imaging ability in the near field to resolve two identical point sources with a $\lambda / 3$ spacing. Although the WGM effect is stronger and the electric field intensity curve at the imaging position shows obvious resonance fluctuation, it slightly affects the resolution imaging effect of the solid immersion square MFEL at the frequency of $11 \mathrm{GHz}$, as shown in Fig. 5(c). Similarly, Figs. 5(a), 5(b), and 5(d) show the electric field distribution at the corresponding frequencies of $9 \mathrm{GHz}, 11 \mathrm{GHz}$, and $12 \mathrm{GHz}$, respectively. From the figures, it shows that solid immersion square MFELs with $n_{0}=2.4$ have a very good ability of super-resolution imaging by capturing large wave-number evanescent wave components at the imaging plane to improve the resolution. Consequently, the solid immersion square MFELs with $n_{0}=$ 2.4 overcome the diffraction limit to achieve super-resolution.

Finally, we discuss further application of the solid immersion square MFEL. The solid immersion square MFEL is cascaded to form a super-resolution information transmission channel for potential optical communication, as shown in Fig. 6. Three identical solid immersion square MFELs with $n_{0}=2.4$ are arranged into a row to build up a transmission channel with an air gap of $0.6 \mathrm{~mm}$ between solid immersion square MFELs. A pair of

(c)

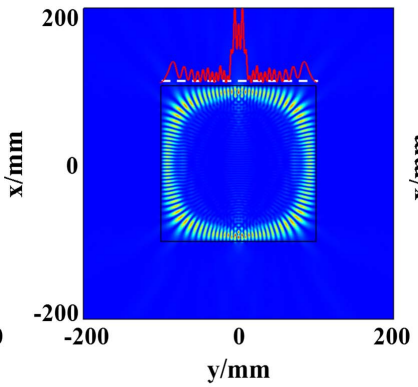

(g)

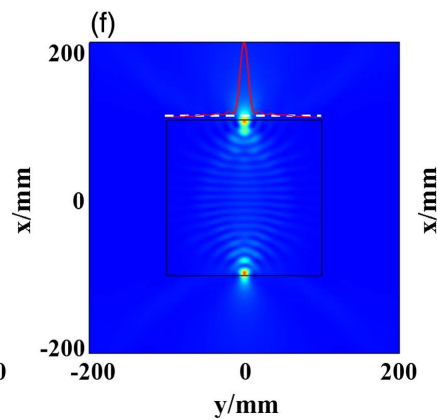

(b)
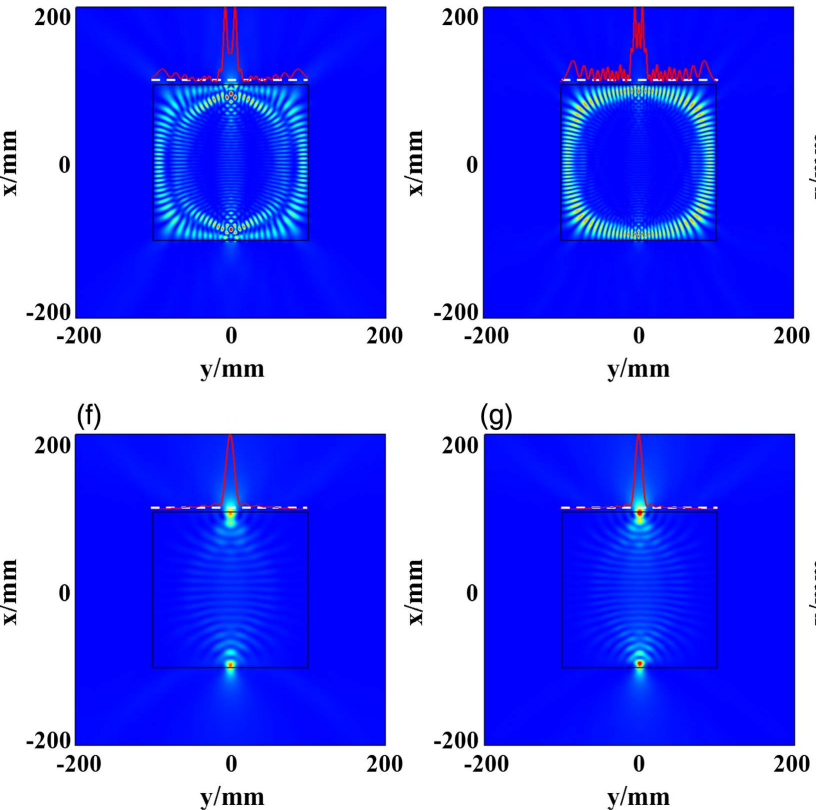

(d)

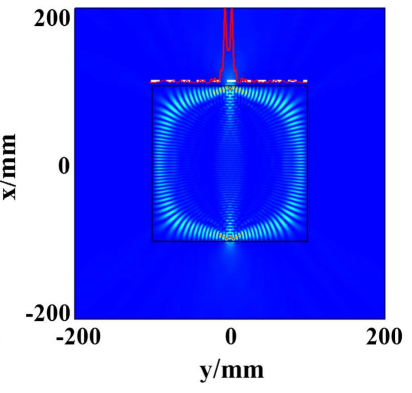

(h)

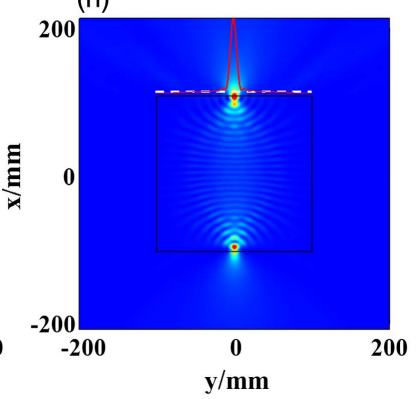

Fig. 5. (a)-(d) Imaging performance of solid immersion square MFELs with $n_{0}=2.4$ at the frequencies of $8 \mathrm{GHz}, 9 \mathrm{GHz}, 11 \mathrm{GHz}$, and $12 \mathrm{GHz}$, respectively. (e)-(g) Imaging performance of conventional square MFELs with $n_{0}=1$ at the frequencies of $8 \mathrm{GHz}, 9 \mathrm{GHz}, 11 \mathrm{GHz}$, and $12 \mathrm{GHz}$, respectively. 


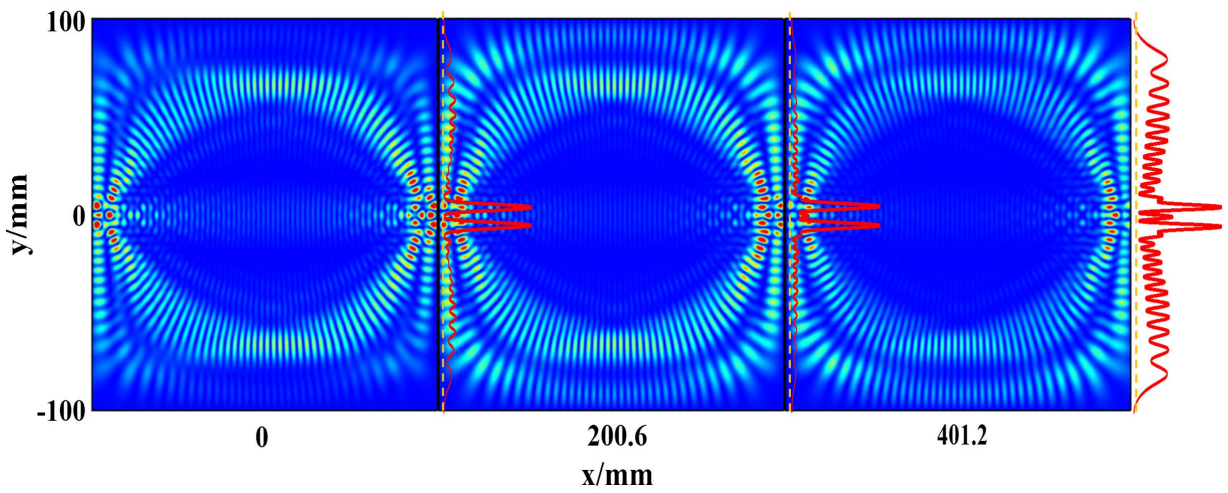

Fig. 6. Electric field distribution of super-resolution information channel cascaded by three identical solid immersion MFELs with a $0.6 \mathrm{~mm}$ air gap at $12 \mathrm{GHz}$.

point sources is placed at $x=-100 \mathrm{~mm}$ with a spacing of $\lambda / 3$ to excite TE waves at the frequency of $12 \mathrm{GHz}$. The red curve describes the electric field intensity at different imaging planes with $y=-100$ to $100 \mathrm{~mm}$. The size of the air gap in the transmission channel composed of solid immersion square MFELs is determined by referring to the numerically calculated results, as shown in Fig. S1 of Supplementary Material. When only one point source is placed in front of the super-resolution information transmission channel, through the observation of the imaging performance of three solid immersion square MFELs, it can be easily found that the information transmission effect and super-resolution performance of the designed super-resolution information transmission channel are relatively good when the air gap spacing is $0.6 \mathrm{~mm}$, as shown in Fig. S1 of Supplementary Material. Generally, when the image point is far away from the source point, the resolution effect will become worse. However, according to the electric field intensity distribution in Fig. 6, it can be seen that even if the distance between the imaging point and source point is increased, the solid immersion square MFELs can still overcome the diffraction limit within a certain range and maintain a good ability of super-resolution imaging. The evanescent wave component with large wave number can be collected at the imaging plane to improve the resolution. Based on the solid immersion square MFEL's super-resolution effect, we design the channel for super-resolution imaging information transmission with larger capacity, which has a good prospect of optical communication applications.

Although some techniques such as spatial drilling ${ }^{[41-43]}$ and gray-scale electron-beam lithography (EBL) techniques ${ }^{[4]}$ can be used to fabricate GRIN lenses, it is currently difficult to find materials with RI of 4.5 in the visible or infrared (IR) region to achieve the designed solid immersion square MFEL. With the development of the 3D gradient glass technology ${ }^{[45,46]}$, there is still a great possibility to achieve it.

\section{Conclusion}

In summary, the solid immersion circular MFEL is transformed into a solid immersion square MFEL using SC mapping. The solid immersion square MFEL maintains the super-resolution imaging characteristics of the original circular MFEL and, at the same time, can overcome the disadvantage of a curved surface, which may pave the way for further applications such as real-time imaging, in vivo imaging, and photolithography. Through simulated calculations, the designed square MFEL can achieve super-resolution imaging at most frequencies. Moreover, the cascading solid immersion square MFELs can effectively transmit super-resolution information and have a good prospect of optical communication applications. With the development of $3 \mathrm{D}$ printing technology ${ }^{[45,46]}$, the solid immersion MFEL is expected to be fabricated in the high-frequency band, such as terahertz $(\mathrm{THz}), \mathrm{IR}$, or even visible frequencies.

\section{Acknowledgement}

This work was supported by the National Key Research and Development Program of China (No. 2020YFA0710100), National Natural Science Foundation of China (Nos. 92050102 and 11874311), and Fundamental Research Funds for the Central Universities (No. 20720200074).

\section{References}

1. J. B. Pendry, "Negative refraction makes a perfect lens," Phys. Rev. Lett. 85, 3966 (2000).

2. N. Fang, H. Lee, S. Cheng, and X. Zhang, "Sub-diffraction-limited optical imaging with a silver superlens," Science 308, 534 (2005).

3. T. Taubner, D. Korobkin, Y. Urzhumov, G. Shvets, and R. Hillenbrand, "Near-field microscopy through a SiC superlens," Science 313, 1595 (2006).

4. I. I. Smolyaninov, Y. J. Hung, and C. C. Davis, "Magnifying superlens in the visible frequency range," Science 315, 1699 (2007).

5. X. Zhang and Z. Liu, "Superlenses to overcome the diffraction limit," Nat. Mater. 7, 435 (2008).

6. T. Huang, L. Yin, J. Zhao, C. Du, and P. Liu, “Amplifying evanescent waves by dispersion-induced plasmons: defying the materials limitation of the superlens," ACS Photon. 7, 2173 (2020).

7. Z. Liu, H. Lee, Y. Xiong, C. Sun, and X. Zhang, "Far-field optical hyperlens magnifying sub-diffraction-limited objects," Science 315, 1686 (2007).

8. J. Rho, Z. Ye, Y. Xiong, X. Yin, Z. Liu, H. Choi, G. Bartal, and X. Zhang, "Spherical hyperlens for two-dimensional sub-diffractional imaging at visible frequencies," Nat. Commun. 1, 143 (2010).

9. D. A. Fletcher, K. B. Crozier, K. W. Guarini, S. C. Minne, G. S. Kino, C. F. Quate, and K. E. Goodson, "Microfabricated silicon solid immersion lens,” J. Microelectromech. Syst. 10, 450 (2001). 
10. F. M. Huang, N. Zheludev, Y. Chen, and F. Javier Garcia de Abajo, "Focusing of light by a nanohole array," Appl. Phys. Lett. 90, 091119 (2007).

11. D. R. Mason, M. V. Jouravlev, and K. S. Kim, "Enhanced resolution beyond the Abbe diffraction limit with wavelength-scale solid immersion lenses," Opt. Lett. 35, 2007 (2010).

12. M. S. Kim, T. Scharf, M. T. Haq, W. Nakagawa, and H. P. Herzig, "Subwavelength-size solid immersion lens," Opt. Lett. 36, 3930 (2011).

13. A. Bogucki, Ł. Zinkiewicz, M. Grzeszczyk, W. Pacuski, K. Nogajewski, T. Kazimierczuk, A. Rodek, J. Suffczyński, K. Watanabe, and T. Taniguchi, "Ultra-long-working-distance spectroscopy of single nanostructures with aspherical solid immersion microlenses," Light Sci. Appl. 9, 48 (2020).

14. H. Zhu, W. Fan, S. Zhou, M. Chen, and L. Wu, "Polymer colloidal spherebased hybrid solid immersion lens for optical super-resolution imaging," ACS Nano 10, 9755 (2016).

15. F. Wen, B. Yan, Z. Wang, and L. Wu, "Three-dimensional all-dielectric metamaterial solid immersion lens for subwavelength imaging at visible frequencies," Sci. Adv. 2, e1600901 (2016).

16. A. Novitsky, T. Repän, R. Malureanu, O. Takayama, E. Shkondin, and A. V. Lavrinenko, "Search for superresolution in a metamaterial solid immersion lens," Phys. Rev. A 99, 023835 (2019).

17. R. K. Luneburg, Mathematical Theory of Optics (University of California, 1964).

18. A. L. Mikaelian and A. M. Prokhorov, "V self-focusing media with variable index of refraction," Prog. Opt. 17, 279 (1980).

19. N. Kundtz and D. R. Smith, "Extreme-angle broadband metamaterial lens," Nat. Mater. 9, 129 (2010).

20. U. Leonhardt and T. G. Philbin, "Perfect imaging with positive refraction in three dimensions," Phys. Rev. A 81, 011804(R) (2010).

21. H. F. Ma and T. J. Cui, "Three-dimensional broadband and broad-angle transformation-optics lens," Nat. Commun. 1, 124 (2010).

22. T. Tyc, L. Herzánová, M. Šarbort, and K. Bering, "Absolute instruments and perfect imaging in geometrical optics," New J. Phys. 13, 115004 (2011).

23. Y. Zhao, Y. Zhang, M. Zheng, X. Dong, X. Duan, and Z. Zhao, "Threedimensional Luneburg lens at optical frequencies," Laser Photonics Rev. 10, 665 (2016).

24. S. Tao, Y. Zhou, and H. Chen, "Maxwell's fish-eye lenses under SchwartzChristoffel mappings," Phys. Rev. A 99, 013837 (2019).

25. Q. Wu, J. P. Turpin, and D. H. Werner, "Integrated photonic systems based on transformation optics enabled gradient index devices," Light Sci. Appl. 1, e38 (2012).

26. S. Li, Y. Zhou, J. Dong, X. Zhang, E. Cassan, J. Hou, C. Yang, S. Chen, D. Gao, and $\mathrm{H}$. Chen, "Universal multimode waveguide crossing based on transformation optics," Optica 5, 1549 (2018).

27. M. G. Scopelliti and M. Chamanzar, "Ultrasonically sculpted virtual relay lens for in situ microimaging," Light Sci. Appl. 8, 65 (2019).
28. C. He, J. Chang, Q. Hu, J. Wang, J. Antonello, H. He, S. Liu, J. Lin, B. Dai, D. S. Elson, P. Xi, H. Ma, and M. J. Booth, "Complex vectorial optics through gradient index lens cascades," Nat. Commun. 10, 4264 (2019).

29. A. Forbes, "Common elements for uncommon light: vector beams with GRIN lenses," Light Sci. Appl. 8, 111 (2019).

30. Y. Zhang, Y. He, H. Wang, L. Sun, and Y. Su, "Ultra-broadband mode size converter using on-chip metamaterial-based Luneburg lens," ACS Photon. 8, $202(2020)$

31. J. C. Maxwell, "Solutions of problems," Cambridge Dublin Math. J. 8, 188 (1854).

32. X. Wang, H. Chen, H. Liu, L. Xu, C. Sheng, and S. Zhu, "Self-focusing and the Talbot effect in conformal transformation optics," Phys. Rev. Lett. 119, 033902 (2017).

33. J. Chen, Y. Zhou, H. Chu, Y. Lai, H. Chen, M. Chen, and D. Fang, "Highly efficient gradient solid immersion lens with large numerical aperture for broadband achromatic deep subwavelength focusing and magnified far field," Adv. Opt. Mater. 9, 2100509 (2021).

34. Y. Zhou, Z. Hao, P. Zhao, and H. Chen, "Super-resolution imaging in absolute instruments," arXiv:2107.01632 (2021).

35. J. B. Pendry, D. Schurig, and D. R. Smith, "Controlling electromagnetic fields," Science 312, 1780 (2006).

36. U. Leonhardt, "Optical conformal mapping," Science 312, 1777 (2006).

37. H. Chen, C. T. Chan, and P. Sheng, "Transformation optics and metamaterials," Nat. Mater. 9, 387 (2010).

38. H. Chen and W. Xiao, "Morse lens," Chin. Opt. Lett. 18, 062403 (2020).

39. M. Schmiele, V. S. Varma, C. Rockstuhl, and F. Lederer, "Designing optical elements from isotropic materials by using transformation optics," Phys. Rev. A 81, 033837 (2010).

40. U. Leonhardt and T. G. Philbin, Geometry and Light: The Science of Invisibility (Courier Corporation, 2010).

41. J. Valentine, J. Li, T. Zentgraf, G. Bartal, and X. Zhang, "An optical cloak made of dielectrics, "Nat. Mater. 8, 568 (2009).

42. D. Headland, A. K. Klein, and M. Fujita, "Dielectric slot-coupled halfMaxwell fisheye lens as octave-bandwidth beam expander for terahertzrange applications," arXiv:2101.11210 (2021).

43. L. Zhang, L. Wang, Y. Wu, and R. Tai, "Plasmonic Luneburg lens and plasmonic nano-coupler," Chin. Opt. Lett. 18, 092401 (2020).

44. O. Bitton, R. Bruch, and U. Leonhardt, "Two-dimensional Maxwell fisheye for integrated optics," Phys. Rev. Appl. 10, 044059 (2018).

45. C. R. Ocier, C. A. Richards, D. A. Bacon-Brown, Q. Ding, R. Kumar, T. J. Garcia, J. van de Groep, J.-H. Song, A. J. Cyphersmith, A. Rhode, A. N. Perry, A. J. Littlefield, J. Zhu, D. Xie, H. Gao, J. F. Messinger, M. L. Brongersma, K. C. Toussaint, L. L. Goddard, and P. V. Braun, "Direct laser writing of volumetric gradient index lenses and waveguides," Light Sci. Appl. 9, 196 (2020).

46. R. Dylla-Spears, T. D. Yee, K. Sasan, D. Nguyen, N. A. Dudukovic, J. M. Ortega, M. A. Johnson, O. D. Herrera, F. J. Ryerson, and L. L. Wong, "3D printed gradient index glass optics," Sci. Adv. 6, 7 (2020). 